\title{
Prévention et dépistage endoscopiques du cancer colo-rectal : toujours du nouveau
}

Malgré la multiplication, ces quinze dernières années dans les pays occidentaux, des examens endoscopiques du côlon - notamment par coloscopie totale - , on ne sait si une telle pratique a déjà fait diminuer la mortalité par cancer colo-rectal, si forte dans ces pays. En dehors du problème posé par les sujets symptomatiques, ou asymptomatiques mais jugés à risque - par exemple porteurs de maladies rectocoliques inflammatoires ou ayant un contexte familial patent de cancer recto-colique -, nos idées sur la prévention ou le dépistage endoscopique du cancer recto-colique chez les sujets asymptomatiques ne sont toujours pas claires. Certaines institutions, nordaméricaines (National Cancer Institute, American Gostroenterological Association, American Cancer Society, American College of Physicians) ou internationales (Centre de collaboration pour la prévention du cancer colo-rectal de l'OMS), ont conseillé la pratique, tous les trois à cinq ans à partir de 50 ans, d'une coloscopie au moins sigmoïdienne associée à la recherche annuelle de sang occulte dans le selles; d'autres ont considéré qu'il n'y avait pas encore assez de données pour recommander un dépistage des sujets asymptomatiques et non à risque [1], ou que, chez ceux-ci, une seule coloscopie totale à partir de 60 ans suffisait [2].

Deux articles récents sont d'un grand intérêt potentiel dans ce domaine. Le premier [3] apporte, dans une étude cas témoins, de solides arguments en faveur de la sigmoïdoscopie de prévention-dépistage. Il a déterminé la fréquence avec laquelle 261 sujets décédés de cancer rectal ou sigmoïdien, de 1971 à 1988 , avaient subi une sigmoïdoscopie (au tube rigide !) dans les dix ans (sans symptômes) ayant précédé le diagnostic, par comparaison à la fréquence relevée chez 868 témoins appariés pour l'âge et le sexe : une ou plusieurs sigmoïdoscopies de dépistage réduisaient le risque de décès par cancer rectal ou du côlon distal de 60 à que ce risque était déjà significativement réduit, pour une période d'au moins dix ans, après un seul examen; bien entendu le bénéfice apparent, sur la mortalité, tiré de l'endoscopie se limitait au segment d'intestin (en l'occurrence le recto-sigmoïde) examiné dans ce travail.

Un autre travail [4], apporte des faits nouveaux sur l'histoire naturelle des polypes adénomateux du sigmoïde et du rectum : il a évalué le risque, à long terme, de cancer colo-rectal, après une première polypectomie colo-rectale distale pour adénome, chez 1618 patients suivis en moyenne pendant 13,8 ans. Globalement, le risque sur le rectum dans cette population n'était pas différent de celui de la population générale, bien que dix fois plus élevé chez les femmes que chez les hommes : 12 des 14 sujets qui ont développé un cancer du rectum avaient un adénome initial en dysplasie au moins moyenne, de diamètre supérieur à $1 \mathrm{~cm}$ et dont l'excision avait été jugée le plus souvent insuffisante. Le risque de cancer colique, quant à lui, était corrélé au type histologique, au diamètre et au nombre d'adénomes dans le recto-sigmoïde initialement exploré : parmi 842 malades ayant un adénome recto-sigmoïdien de type tubulo-villeux ou villeux, et/ou d'un diamètre de plus de $1 \mathrm{~cm}$, un cancer colique est survenu dans 31 cas; le risque défini par le rapport d'incidence standardisé (cas observés/cas attendus) a été globalement de 3,6 (2,4 à 5), et de $6,6(3,3$ à 11,8$)$ s'il y avait des adénomes multiples. Parmi les 776 autres patients ayant de petits $(<1 \mathrm{~cm})$ adénomes tubuleux, uniques ou multiples, un cancer colique n'est survenu que dans 4 cas, donnant un rapport d'incidence standardisé de $0,5(0,1$ à 1,3$)$. Ce travail suggère très clairement que le risque ultérieur de cancer rectocolique est faible et non différent de celui de la population générale chez les sujets ayant eu un seul adénome, de type tubuleux et de moins de $1 \mathrm{~cm}$, dans le rectum ou le sigmoïde. Or, actuellement, dès qu'un adénome recto-sigmoïdien est identifié et enlevé, une colonoscopie totale de surveillance est habituellement recommandée. On conçoit que les résultats d'Atkin et al. [4], qui rejoignent ceux d'un travail antérieur [5] mais demandant confirmation, puissent avoir d'importantes conséquences pratiques.

Tous ces faits semblent militer, en matière de prévention et de dépistage endoscopiques de la population générale asymptomatique, pour la coloscopie au moins sigmoïdienne avec une périodicité de cinq ans. Le problème est moins simple à l'échelon individuel : chez le sujet symptomatique et consultant, il ne paraît guère possible de proposer autre chose qu'une coloscopie totale ; de plus, la périodicité de celle-ci est certainement à réduire dès qu'il s'agit de sujets à risque, ayant par exemple une histoire familiale de cancer colo-rectal chez un ou plusieurs parents du premier degré. Finalement, même s'ils ne sont valables que pour les méthodes diagnostiques employées et pour la zone de côlon initialement étudiée, ces résultats $[3,4]$ contribuent à l'approche d'un usage optimal des techniques endoscopiques de prévention et de dépistage.

C. M.

1. Winawer SJ, Schottenfeld D, Flehinger BJ Colorectal cancer screening. $J$ Natl Cancer Inst $1991 ; 83: 243-53$.

2. Levin B. Screening sigmoidoscopy for colorectal cancer. N Engl J Med 1992 ; 326 : 700-2.

3. Selby JV, Friedman GD, Quesenberry CP, Weiss NS. A case-control study of screening sigmoidoscopy and mortality from colorecta cancer. N Engl J Med 1992 ; 326 : 653-7.

4. Atkin WS, Morson BC, Cuzick J. Longterm risk of colorectal cancer after excision of rectosigmoid adenomas. N Engl J Med 1992 ; 326 : 658-62.

5. Grossman S, Milos ML, Tekawa IS, Jewell NP. Colonoscopic screening of persons with suspected risk factors for colon cancer. II. Past history of colorectal neoplasms. Gastroenterology $1989 ; 96$ : 299-306. 